\title{
Surface and Antibacterial Properties of Titanium Dioxide Nanoparticles Synthesized in Plasma Discharge under Ultrasonic Cavitation
}

\author{
Nikolay Bulychev ${ }^{1,2}$, Svetlana Dimitrieva ${ }^{1,3}$, Irina Kozerozhets $^{4,5}$, Oksana Kuznetsova ${ }^{6}$, \\ Igor Nikitin ${ }^{3}$ \\ ${ }^{1}$ P.N. Lebedev Physical Institute of Russian Academy of Sciences, Leninsky pr-t, 53, Moscow, \\ Russian Federation \\ ${ }^{2}$ Moscow Aviation Institute (National Research University), Volokolamskoe shosse, 4, Moscow, \\ Russian Federation \\ ${ }^{3}$ K.G. Razumovsky Moscow State University of technologies and management \\ (The First Cossack University), St. Zemlyanoy Val, 73, Moscow, Russian Federation \\ ${ }^{4}$ N.S. Kurnakov Institute of General and Inorganic Chemistry of Russian Academy of Sciences, \\ Leninsky pr-t, 31, Moscow, Russian Federation \\ ${ }^{5}$ National University of Science and Technology MISIS, Leninsky pr-t, 4, Moscow, Russian \\ Federation \\ ${ }^{6}$ V.M. Gorbatov Federal Research Center for Food Systems of Russian Academy of Sciences, \\ Talalikhina str., 26, Moscow, Russian Federation
}

\begin{abstract}
In this work, it is shown that intensive treatment of dispersed systems with ultrasound is an effective method for enhancing the interaction of particles and polymer with the subsequent formation of adsorption layers of high thickness and strength. This makes possible to obtain highly stable dispersed systems with a modified surface of the solid phase. The studies proved the important role of intensive ultrasonic action for controlling the properties of the nanoparticles surface in heterophase systems. It is shown that an increase in the activity of the surface of nanoparticles as a result of ultrasonic treatment is of great importance for the study of their antibacterial activity: after ultrasonic activation, nanoparticles are more active in interaction with cell membranes.
\end{abstract}

\section{Introduction}

Modern food systems cover a huge number of links from "field" to "consumer" and represent the environment for the emergence, transmission and propagation of infectious diseases. Numerous microorganisms and viruses can circulate, mutate and be transmitted to humans with food. Foodborne infections are rising increasingly. Since 2017, there has been a progressive increase in infectious foodborne diseases in the EU countries. Anthropogenic effects impact on the environment accelerating the evolution of microbes and among the traditional contaminants of food raw materials and food products, lead to the emergence of 
bacteria with altered properties resistant to environmental influences, antibiotics, etc., with additional pathogenic factors, which currently explains an increasing number of so-called "emergent" (unexpected) infections from food. According to the WHO data, the group of such diseases includes about two hundred infectious diseases that have spread in the world recently, while $75 \%$ of emerging infections are diseases of zoogenic origin.

Measures for disinfection and antimicrobial processing of food products and equipment require high energy costs and the use of a large number of reagents and equipment, which increases the cost of food production and reduces the energy efficiency of the agroindustrial complex.

This shows that new antimicrobial materials and technologies are needed to create such materials as an effective safety tool in the food industry, limit the use of antimicrobial agents and reduce the risks of threats associated with the propagation of foodborne infections. Nanomaterials of various compositions occupy an important place among such promising materials. Therefore, the development of methods for obtaining such materials and the study of their properties is an urgent scientific problem.

In previous studies, dispersed systems of micro- and nanoparticles obtained as a result of intensive action of various types have been studied in detail [1-3]. It was found that mechanical activation significantly affects the properties of dispersed systems, significantly increasing their stability and dispersion of the solid phase, and also affects the properties of the particle surface, causing the formation of active centers that can participate in the interaction with (bio) -organic and polymer molecules, matrices etc.

Therefore, an important task is to study the effect of intensive ultrasonic action on the synthesis of bactericidal nanoparticles, as well as to obtain quantitative information about the adsorption polymer layers formed during this action as an informative method for studying the properties of the surface of nanoparticles and its activity and reactivity. Thus, titanium dioxide nanoparticles were synthesized for detailed studies, including because it is known from the literature about their bactericidal properties (see, for example, [5, 6]).

$\mathrm{TiO} 2$ nanoparticles and their aggregates can attach to the cell membrane and interact with surface receptors, including those involved in phagocytosis and micropinocytosis, which leads to the introduction of nanoparticles into cells. Mechanical stress due to the interaction of cells with nanoparticles can disrupt the integrity of the cell membrane and affect ion homeostasis and the activity of membrane-bound receptors and enzymes. $\mathrm{TiO} 2$ particles with a primary particle size of $\sim 40 \mathrm{~nm}$ interact to a significant extent with phospholipid layers $[5,6]$. The aggregation of these particles initially has little effect on this interaction, but long-term aggregation affects the interaction, as a result, the aggregates penetrate the lipid layer rather than being adsorbed on the surface. Larger $\mathrm{TiO} 2$ particles interact less strongly with phospholipid layers. $\mathrm{TiO} 2$ nanoparticles not bound to the cell membrane can also photocatalytically generate reactive oxygen species (most notably hydrogen peroxide) that contribute to membrane damage. Being introduced into the cell, $\mathrm{TiO} 2$ nanoparticles are transported by phagosomes into lysosomes, causing lysosomal stress and damage, accumulation of nanoparticles in multilayer vesicles obtained from lysosomes, and ultimately released into the cytosol, where it can interact with various cellular components. Intracellular accumulation of $\mathrm{TiO} 2$ nanoparticles leads to DNA damage, changes in DNA conformation due to the binding of nanoparticles, and ultimately to cell death. 


\section{Material and methods}

Titanium dioxide nanoparticles were synthesized in a plasma discharge under the action of ultrasonic cavitation in an aqueous medium as described elsewhere [7]. Titanium rods were used as starting materials. The resulting nanoparticles were studied by dynamic light scattering, electrokinetic potential measuring and electron microscopy using Malvern Zetasizer Nano and Carl Zeiss Evo 50 instruments. For surface activity studies, nanoparticles were treated by ultrasound: duration 2 minutes, exposure frequency $20 \mathrm{kHz}$ and specific power $0.1-0.2 \mathrm{~W} / \mathrm{cm} 3$ before the cavitation and $1-1.5 \mathrm{~W} / \mathrm{cm} 3$ in the developed cavitation regime.

\section{Results and discussion}

The small size and high aggregative stability of nanoparticles made it possible to obtain a relatively stable aqueous dispersed system without the use of any stabilizers. In the case of aggregation of nanoparticles, they were ultrasonically dispersed for $1 \mathrm{~min}$.

Using the dynamic light scattering method, the particle size in aqueous dispersed systems and their aggregate stability were estimated. It was shown that $\mathrm{TiO} 2$ nanoparticles were obtained with a quite narrow size distribution and a distribution peak was in the region of 40-50 nm. The sedimentation stability of the obtained samples is maintained for about 1.5-2 months. Particle size data are shown in Fig. 1 and 2.

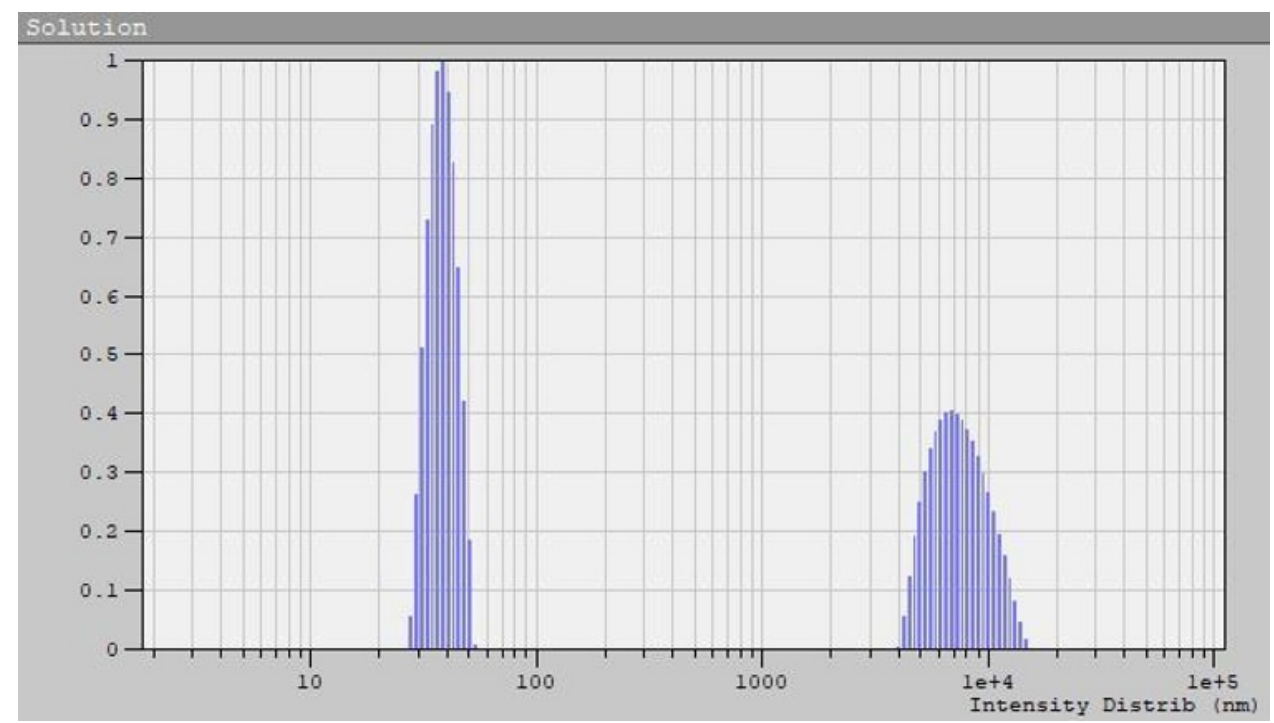

Fig. 1. Dynamic light scattering data for $\mathrm{TiO} 2$ nanoparticles. 


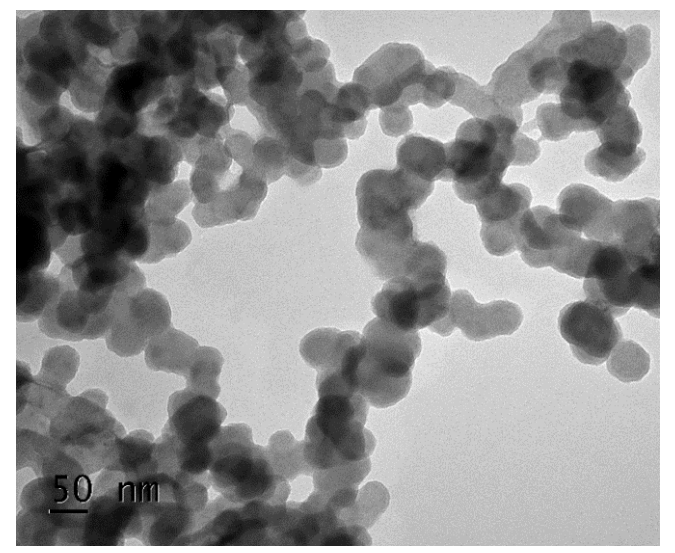

Fig. 2. TEM visualization of synthesized $\mathrm{TiO} 2$ nanoparticles.

Electron microscopy examination showed that, first, spherical particles were obtained, and second, during aggregation they did not enlarge in size, but form composite aggregates. This gives the possibility to work with them after ultrasonic treatment of the suspension.

To study the properties of the particle surface, titanium dioxide nanoparticles in a plasma discharge were obtained by three methods - without application of ultrasound, with ultrasound before the cavitation, and with ultrasound in the cavitation regime. Accordingly, the interaction of the surface of the obtained nanoparticles with polymers was carried out both by means of conventional adsorption and by means of mechanical activation of the surface of nanoparticles during the treatment of dispersed systems in an ultrasonic field before the cavitation and in the regime of developed cavitation. The processing parameters and their effect on the properties of the resulting dispersed systems were optimized earlier [3]. The efficiency of modification of the particles surface with polymers was examined by measuring the electrokinetic potential of the surface ( $\xi$-potential), its change characterizes the thickness of the adsorbate surface layer [8-13]. A water-soluble polymer, a cellulose derivative ethyl hydroxyethyl cellulose (EHEC), was chosen as a surface-active polymer.

Fig. 3 shows the dependences of the surface potential of titanium dioxide nanoparticles on the relative concentration of the polymer.

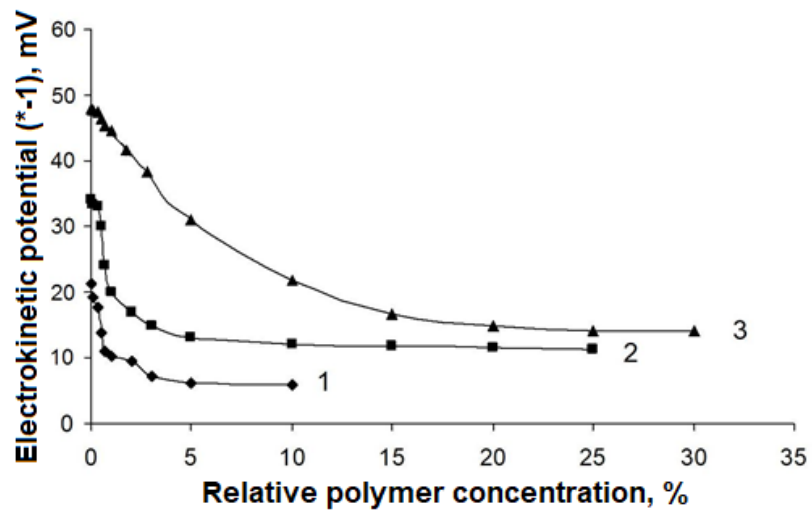

Fig. 3. Dependence of the surface potential of titanium dioxide nanoparticles in a $1 \%$ aqueous dispersed system on the relative concentration of the polymer stabilizer ethyl hydroxyethyl cellulose (EHEC). 1 - without mechanical treatment of the system, 2 - after ultrasonic treatment before the cavitation, 3 - after ultrasonic treatment in the cavitation regime. 
From Fig. 3 one can infer, that the values of the $\xi$-potential of titanium dioxide nanoparticles without polymer, synthesized in the absence and in the presence of ultrasonic action, differ drastically. Without ultrasound, the initial value of the $\xi$-potential is $-21 \mathrm{mV}$, while when using ultrasonic action, this value sharply increases and is $-47 \mathrm{mV}$. As the polymer is added to the system, its adsorption on the surface of the particles starts and the value of the $\xi$ potential decrease. Initially, the change of the $\xi$-potential is fast, then it slows down and soon reaches a more or less constant value, which corresponds to the saturation of the adsorption layer. The course of polymolecular adsorption depends on many factors, including the nature of particles and polymer, and can be monitored be the change in the values of the $\xi$-potential upon further addition of the polymer to the system.

The dynamics of the change in the values of the $\xi$-potential of the particles with an increase in the polymer concentration makes it possible to judge about the process of polymer adsorption on the particles surface. Important parameters here are the values of the saturation concentration of the adsorption layer, the rate of its approaching, i.e., the slope of the curve at the initial section, as well as the values of the $\xi$-potential at the initial moment, i.e., in the absence of polymer in the system and that at the moment of saturation of the adsorption layer. Comparison of these values for different systems gives the possibility to draw conclusions about the course of polymer adsorption and the effect of various factors, for example, the presence of intensive mechanical action on the system [1-3]. A further increase in the polymer concentration does not affect the values of the $\xi$-potential of the particles, which indicates the absence of polymolecular adsorption.

At the same time, the difference in the values of the $\xi$-potential at saturation and at the initial moment for the system after ultrasonic exposure is greater, which is a qualitative evidence of the activation of the particle surface, an increase in the amount of adsorbed polymer, and, hence, an increase in the thickness of the adsorption layer.

Thus, comparing the saturation concentrations of adsorption layers for treated and untreated with ultrasound samples, obtained by measuring the $\xi$-potential, we can conclude that intensive mechanical action leads not only to an increase in the stability and dispersion of the system, but also to more active sorption of the polymer on the surface, and hence, to an increase in the amount of adsorbed polymer.

It is important to note that the data obtained about the increase in the surface activity of nanoparticles as a result of ultrasonic treatment are of great importance for the study of their antibacterial activity: it is obvious that, being more active when interacting with organic polymers, nanoparticles after ultrasonic activation will also be more active in interacting with cell membranes.

To examine the bactericidal activity of nanoparticles, Escherichia coli were used as test microorganisms to evaluate the bactericidal activity against gram-negative bacteria and Staphylococcus aureus was used to evaluate the bactericidal activity against gram-positive bacteria. Disco-diffusion and plate methods were used to examine the nanoparticles. The disk-diffusion method for determining the sensitivity is based on the ability of substances with antibacterial activity to diffuse from paper disks impregnated with them into the nutrient medium, inhibiting the growth of microorganisms seeded on the agar surface.

A standard inoculum was used, corresponding in density to $0.5 \mathrm{McF}$ arland standard and containing approximately $1.5 * 108 \mathrm{CFU} / \mathrm{ml}$. The standard inoculum was pipetted onto the surface of a Petri dish with a nutrient medium in a volume of 1-2 $\mathrm{ml}$, evenly distributed over the surface by shaking, after which the excess inoculum was removed with a pipette. The agar dishes were dried at room temperature for 10-15 minutes. No later than 15 min after inoculation, discs with substances with antibacterial activity (synthesized 
nanoparticles and control) were applied to the surface of the nutrient medium. Standard ampicillin $(1 \mathrm{mg} / \mathrm{ml})$ was used as a positive control. After the application of the discs, the Petri dishes were placed in a thermostat upside down and incubated at $37^{\circ} \mathrm{C}$ for $24 \mathrm{~h}$, after which the diameter of the obtained zone of inhibition was measured. The experiments were repeated three times with similar results.

The width of the zone of inhibition around $\mathrm{TiO} 2$ nanoparticles was $12 \mathrm{~mm}$ for E. coli, and $10 \mathrm{~mm}$ for $\mathrm{S}$. aureus. The diameter of the inhibition zone of $\mathrm{S}$. aureus is smaller than that of E. coli, presumably due to structural differences in the cell wall of gram-positive and gram-negative bacteria. In gram-positive bacteria, such as S. aureus, there is a hard layer of peptidoglycan in the cell wall, which increases the resistance of the microbial cell to the titanium ion, while the E. coli cell membrane is relatively thin and mainly consists of peptidoglycan, and the outer layer consists of lipopolysaccharide, phospholipids and a lipoprotein layer with a tendency to adhere titanium ion to the cell membrane. Therefore, $\mathrm{TiO} 2$ nanoparticles exhibited better antibacterial activity against E. coli than against S. aureus.

\section{Conclusions}

Thus, nanosized particles of titanium oxide were obtained and characterized. The possibilities of controlling the activity of the surface of nanoparticles by changing the intensity of ultrasonic action are shown. The interaction of the surface of nanoparticles with molecules of organic polymers was studied by measuring the electrokinetic potential of the surface of nanoparticles. It is shown that ultrasonic action activates the interaction of the polymer and the surface. Studies of the antibacterial activity of nanoparticles in contact with microorganisms confirmed the bactericidal properties of nanoparticles.

\section{Acknowledgements}

This work was supported by Ministry of Science and Higher Education of the Russian Federation, project No. 2020-1902-01-288 (Agreement No. 075-15-2020-775).

\section{References}

1. N. Bulychev, K. Dirnberger, H. Reimann, C. Schaller, T. Schauer, V. Zubov, C.D. Eisenbach, European Coatings Journal. 3 (32), 34-37 (2007)

2. N. Bulychev, O. Confortini, P. Kopold, K. Dirnberger, T. Schauer, F.E. Du Prez, V. Zubov, C.D. Eisenbach, Polymer. 48 (9), 2636-2643 (2007)

3. N. Bulychev, K. Dirnberger, I. Arutunov, P. Kopold, T. Schauer, V. Zubov, C.D. Eisenbach, Progress in Organic Coatings. 62 (3), 299-306 (2008)

4. N.A. Bulychev, Inorganic Materials. 46, 4, 393-398 (2010)

5. Z. Luo, Z. Li, Z. Xie, I.M. Sokolova, L. Song, W.J.G.M. Peijnenburg, M. Hu, Y. Wang, Nano-micro Small. 16, 2002019 (2020)

6. A. Vakurov, R. Drummondbrydson, O. Ugwumsinachi, A. Nelson, J. Colloid Interface Sci. 473, 75 (2016) 
7. N.A. Bulychev, M.A. Kazaryan, M.N. Kirichenko, A.V. Ivanov, International Journal of Nanotechnology. 16, 1/2/3, 34-41 (2019)

8. C. Schaller, T. Schauer, K. Dirnberger, C.D. Eisenbach. Eur. Phys. J., E 6, 365-376 (2001)

9. C. Schaller, A. Schoger, K. Dirnberger, T. Schauer, C.D. Eisenbach, Macromol. Symp. 179, 173-188 (2002)

10. H. Maier, J.A. Baker, J.C. Berg, J. Colloid Interface. Sci. 119, 512-517 (1987)

11. N.P. Miller, J.C. Berg, Colloids and Surfaces. 59, 119-128 (1991)

12. M.L. Carasso, W.N. Rowlands, R.W. O’Brien, J. Colloid Interface Sci. 193, 200-214 (1997)

13. R.W. O’Brien, Part. Syst. Charact. 19, 1-9 (2002) 